\title{
The role of tomato and corn root exudates on Glomus mosseae spores germination and Ralstonia solanacearum growth in vitro.
}

\begin{abstract}
An in vitro experiment was conducted to study the effect of different plant root exudates on germination of Glomus mosseae and the growth of bacterial wilt Ralstonia solanacearum. Mycorrhizal spore germination increased when the volume of Mycorrhizal Tomato Root Exudates (MTRE) increased and in contrast, a negative relationship was recorded when the volume of Non-Mycorrhizal Tomato Root Exudates (NMTRE) increased. Similarly, the Mycorrhizal Corn Root Exudates (MCRE) was able to increase the percentage of germinated spores as compared to the Non-Mycorrhizal Corn Root Exudates (NMCRE). The antagonistic effect between Ralstonia solanacearum and Glomus mosseae was also studied in this research. There was no inhibition effect of mycorrhizal and non-mycorrhizal tomato and corn root exudates on growth of R. solanacearum. The study indicated that Glomus mosseae spore germination could be influenced by the host plant or $\mathrm{pH}$ medium.
\end{abstract}

Keyword: In vitro; Bacteria; Mycorrhizae; Root exudates; Antagonistic. 